Mathematical Research Letters 4, 35-44 (1997)

\title{
HARMONIC SECTIONS OF POLYNOMIAL GROWTH
}

\section{PETER Li}

In a series of papers [C-M 1-6], Colding and Minicozzi studied the space harmonic functions of polynomial growth and eventually proved a conjecture of Yau $[\mathrm{Y}]$ in $[\mathrm{C}-\mathrm{M}$ 5].

Yau's conjecture. Let $M^{n}$ be a complete manifold with non-negative Ricci curvature. If $\rho(x)$ denotes the distance of any point $x$ to a fixed point $p \in M$, then the space of harmonic functions,

$$
\mathcal{H}_{d}(M)=\left\{f|\Delta f=0,| f \mid(x)=O\left(\rho^{d}(x)\right) \text { as } \rho \rightarrow \infty\right\},
$$

defined on $M$ which has at most polynomial growth of degree $d$ must be finite dimensional for any $d \in \mathbb{R}^{+}$.

In fact, Colding and Minicozzi [C-M 4] announced, and subsequently proved in [C-M 6], a more general statement. They considered complete manifolds satisfying:

(V) The volume comparison condition asserting that if $V_{x}(r)$ is the volume of the geodesic ball centered at $x \in M$ of radius $r$ then

$$
\left(\frac{r^{\prime}}{r}\right)^{\nu} V_{x}(r) \geq V_{x}\left(r^{\prime}\right)
$$

for some constant $\nu>0$ and for all $r \leq r^{\prime}$; and

(P) The Poincaré inequality stating that there exists a constant $C^{\prime}>0$, such that for all $x \in M$ and $r>0$,

$$
\int_{B_{x}(r)} u^{2} \leq C^{\prime} r^{2} \int_{B_{x}(r)}|\nabla u|^{2}
$$

for any $u \in H_{1,2}\left(B_{x}(r)\right)$ satisfying $\int_{B_{x}(r)} u=0$.

It is known that these two conditions are valid on manifolds with non-negative Ricci curvature with $\nu=n$. Using an argument involving the Poincaré inequality and a ball covering lemma, the authors in [C-M 6] proved:

Received December 5, 1996.

Research partially supported by NSF grant DMS-9626310 
Theorem. (Colding-Minicozzi) Let $M^{n}$ be a complete manifold satisfying $(V)$ and $(P)$. Then the dimension of $\mathcal{H}_{d}(M)$ is finite. Moreover, there exists a constant $C\left(n, \nu, C^{\prime}\right)>0$ depending only on $n, \nu$, and $C^{\prime}$ such that

$$
\operatorname{dim} \mathcal{H}_{d}(M) \leq C d^{\nu-1}
$$

Another condition which often occurs in the literature is:

(V') The volume doubling condition asserting that there exists a constant $\eta>0$ such that

$$
2^{\eta} V_{x}(r) \geq V_{x}(2 r)
$$

for all $x \in M$ and $r>0$.

It is easy to see that the volume doubling condition is almost equivalent to condition (V). In fact, (V) implies the volume doubling condition with $\eta=\nu$. Conversely, the volume doubling condition implies that

$$
\left(\frac{2 r^{\prime}}{r}\right)^{\eta} V_{x}(r) \geq V_{x}\left(r^{\prime}\right)
$$

for $r^{\prime} \geq r$. In the same paper [C-M 6], assuming conditions ( $\left.\mathrm{V}^{\prime}\right)$ and (P) instead, the authors also proved that

$$
\operatorname{dim} \mathcal{H}_{d}(M) \leq C d^{\eta}
$$

The order in $d$ in this case is not sharp. The sharp order should be $d^{\eta-1}$, which will be comparable to the case when condition (V) is assumed.

In [C-M 4], the authors also announced, without indicating the proof, that $\mathcal{H}_{d}(M)$ is finite dimensional if $M$ is a minimal submanifold in Euclidean space with Euclidean volume growth. In the same note, they also announced a finite dimensionality result for harmonic sections of polynomial growth of degree at most $d$ on a Hermitian vector bundle with nonnegative curvature over a manifold with non-negative Ricci curvature. Shortly after the author circulated this preprint, Colding-Minicozzi circulated a new preprint [C-M 7] providing the proofs for the minimal submanifold and the harmonic sections cases. In this paper, they also used a form of mean value inequality similar to the present paper. However their argument did not provide the sharp order in $d$.

It is known [G, S-C 1] that conditions ( $\left.\mathrm{V}^{\prime}\right)$ and (P) imply a Harnack inequality for positive harmonic functions. In particular they imply:

(M) The mean value inequality asserting that there exists a constant $\lambda>0$, such that for all $x \in M$ and $r>0$, any non-negative subharmonic function $f$ defined on $M$ must satisfy

$$
\lambda \int_{B_{x}(r)} f \geq V_{x}(r) f(x) .
$$


Also, in view of the above discussion concerning conditions (V) and (V'), it is convenient for us to introduce:

(W) The weak volume comparison condition asserting that there are constants $C_{0}>0$ and $\nu>0$, such that for all $x \in M$ and $r^{\prime} \geq r>0$,

$$
C_{0}\left(\frac{r^{\prime}}{r}\right)^{\nu} V_{x}(r) \geq V_{x}\left(r^{\prime}\right)
$$

The purpose of this note is to give a short proof of the dimension estimate by assuming only (W) and the weaker condition (M). In fact, the argument also works for sections of a vector bundle who square norms are subharmonic. Moreover, the estimate is sharp in the order of $d$.

Theorem 1. Let $M^{n}$ be a complete manifold satisfying conditions $(W)$ and $(M)$. Suppose $E$ is a rank-m vector bundle over $M$. Let $\mathcal{S}_{d}(M, E) \subset \Gamma(E)$ be a linear subspace of sections of $E$, such that, all $u \in \mathcal{S}_{d}(M, E)$ satisfy

(a) $\Delta|u|^{2} \geq 0$, and

(b) $|u|(x) \leq O\left(\rho^{d}(x)\right)$ as $x \rightarrow \infty$.

Then the dimension of $\mathcal{S}_{d}(M)$ is finite. Moreover there exists a constant $C>0$ depending only on $C_{0}$ and $\nu$ such that

$$
\operatorname{dim} \mathcal{H}_{d}(M) \leq m C \lambda d^{\nu-1} .
$$

Note that the assumption $(\mathrm{M})$ is much weaker than $(\mathrm{P})$. For example (see [L 2]), condition (M) follows from condition (W) and a scale invariant Sobolev inequality of the following form:

(S) The Sobolev inequality asserting that there exists constants $C, \mu>2$, such that for $x \in M$ and $r>0$,

$$
\left(\int_{B_{x}(r)} f^{\frac{2 \mu}{\mu-2}}\right)^{\frac{\mu-2}{\mu}} \leq C V_{x}(r)^{-2 / \mu}\left(r^{2} \int_{B_{x}(r)}|\nabla f|^{2}+\int_{B_{x}(r)} f^{2}\right)
$$

for any compactly supported function $f \in H_{1,2}^{c}\left(B_{x}(r)\right)$.

It is also known [S-C 2] that conditions (W) and (P) imply condition (S). On the other hand, Moser's iteration scheme asserts that conditions (W) and $(\mathrm{S})$ imply condition $(\mathrm{M})$.

Due to the fact that conditions (W) and (S) are quasi-isometric invariants, condition (M) holds on manifolds which are quasi-isometric to a manifold with non-negative Ricci curvature. Also conditions (W) and (S) hold [M-S] on $n$ dimensional minimal submanifolds in Euclidean space $\mathbb{R}^{N}$ with Euclidean volume growth. In this case, the distance function $\rho$ is taken to be the extrinsic distance function of $\mathbb{R}^{N}$. Here the Euclidean volume growth is a growth condition for extrinsic balls

$$
V_{p}\left(M \cap B_{p}(r)\right) \leq C r^{n}
$$


for some point $p \in M$.

It is interesting to point out that while a manifold may satisfy conditions (W) and (M), it may not satisfy Liouville property. In particular, when $M$ is a minimal submanifolds in $\mathbb{R}^{N}$ with Euclidean volume growth, it must have finite dimensional bounded harmonic functions according to Corollary 2, but it is not known that there are no non-constant bounded harmonic functions. Another example is $M=\mathbb{R}^{n} \sharp \mathbb{R}^{n}$ for $n \geq 3$. In this case, one can easily see that there are non-constant bounded harmonic functions, but $M$ satisfies conditions (W) and (M). In contrast, the assumptions in [C-M 6], namely conditions ( $\left.\mathrm{V}^{\prime}\right)$ and $(\mathrm{P})$, imply the (strong) Liouville property.

The first corollary of Theorem 1 generalizes the estimate of Colding-Minicozzi. Other corollaries are related to estimating the dimension of harmonic sections with polynomial growth and holomorphic sections over a Kähler manifold.

We would like to point out that there are many other contributions to this subject. The interested reader should refer to a survey article of the author [L 3] for a more detail reference. Throughout this paper, we will denote all constants that depend on $\nu$ and $C_{0}$ generically by $C$.

The author would like to take this opportunity to express his gratitude to Jiaping Wang for suggesting ways to improve our argument.

Lemma 1. Let $K$ be a $k$-dimensional linear space of sections of a rank-m vector bundle $E$ over $M$. Suppose $M$ satisfies conditions $(W)$ and $(M)$. Assume that the square norm of each section $u \in K$ satisfies the differential inequality

$$
\Delta|u|^{2} \geq 0
$$

Let $\left\{u_{i}\right\}_{i=1}^{k}$ be any basis of $K$. Then for $p \in M, r>0$, and any $0<\epsilon<1 / 2$, they must satisfy the estimate

$$
\sum_{i=1}^{k} \int_{B_{p}(r)}\left|u_{i}\right|^{2} \leq m C \lambda \epsilon^{-(\nu-1)} \sup _{u \in\{\langle A, U\rangle\}} \int_{B_{p}((1+\epsilon) r)}|u|^{2},
$$

where the supremum is taken over all $u \in K$ of the form $u=\langle A, U\rangle$ for some unit vector $A=\left(a_{1}, \ldots, a_{k}\right)$ with $U=\left(u_{1}, \ldots, u_{k}\right)$.

Proof. We will estimate $\sum \int_{B_{p}(r)}\left|u_{i}\right|^{2}$ by using a trick employed by the author in [L 1]. Observe that for any $x \in B_{p}(r)$, there exists a subspace $K_{x} \subset K$ which is of at most codimension- $m$ (see Lemma 11 of [L 1]), such that $u(x)=0$ for all $u \in K_{x}$. Hence, by an orthonormal change of basis, we may assume that $u_{i} \in K_{x}$ for $m+1 \leq i \leq k$ and $\sum_{i=1}^{k}\left|u_{i}\right|^{2}(x)=\sum_{i=1}^{m}\left|u_{i}\right|^{2}(x)$. Since $\Delta\left|u_{i}\right|^{2} \geq 0$, if we denote the distance from $p$ to $x$ by $\rho(x)$, then the mean value inequality (M) 
implies that

$$
\begin{aligned}
\sum_{i=1}^{k}\left|u_{i}\right|^{2}(x) & =\sum_{i=1}^{m}\left|u_{i}\right|^{2}(x) \\
& \leq \lambda V_{x}^{-1}((1+\epsilon) r-\rho(x)) \sum_{i=1}^{m} \int_{B_{x}((1+\epsilon) r-\rho(x))}\left|u_{i}\right|^{2} \\
& \leq \lambda V_{x}^{-1}((1+\epsilon) r-\rho(x)) m \sup _{u \in\{\langle A, U\rangle\}} \int_{B_{p}((1+\epsilon) r)}|u|^{2} .
\end{aligned}
$$

However, condition $(\mathrm{W})$ and the fact that $\rho(x) \leq r$ imply that

$$
\begin{aligned}
V_{x}((1+\epsilon) r-\rho(x)) & \geq C_{0}^{-1}\left(\frac{(1+\epsilon) r-\rho(x)}{(1+\epsilon) r+\rho(x)}\right)^{\nu} V_{x}((1+\epsilon) r+\rho(x)) \\
& \geq C_{0}^{-1}\left(\frac{(1+\epsilon) r-\rho(x)}{2(1+\epsilon) r}\right)^{\nu} V_{p}(r) .
\end{aligned}
$$

Hence, substituting into (2) and integrating over $B_{p}(r)$, we have

$$
\begin{aligned}
\sum_{i=1}^{k} \int_{B_{p}(r)}\left|u_{i}\right|^{2} \leq & \frac{C \lambda}{V_{p}(r)} \sup _{u \in\{\langle A, U\rangle\}} \int_{B_{p}((1+\epsilon) r)} u^{2} \\
& \times \int_{B_{p}(r)}\left((1+\epsilon)-r^{-1} \rho(x)\right)^{-\nu} d x .
\end{aligned}
$$

On the other hand, if we define

$$
f(\rho)=\left((1+\epsilon)-r^{-1} \rho\right)^{-\nu},
$$

then

$$
f^{\prime}(\rho)=\nu r^{-1}\left((1+\epsilon)-r^{-1} \rho\right)^{-(\nu+1)} \geq 0,
$$

and

$$
\int_{B_{p}(r)}\left((1+\epsilon)-r^{-1} \rho(x)\right)^{-\nu} d x=\int_{0}^{r} A_{p}(\rho) f(\rho) d \rho .
$$


Let $\alpha=2^{1 / q}$ for some sufficiently large $q \in \mathbb{Z}^{+}$such that $\alpha \leq 1+\epsilon / 2$. Then

$$
\begin{aligned}
\int_{0}^{r} & A_{p}(\rho) f(\rho) d \rho \\
& =\int_{0}^{2^{-1} r} A_{p}(\rho) f(\rho) d \rho+\sum_{i=0}^{q-1} \int_{\alpha^{i} 2^{-1} r}^{\alpha^{i+1} 2^{-1} r} A_{p}(\rho) f(\rho) d \rho \\
& \leq C V_{p}(r)+\sum_{i=0}^{q-1}\left(f\left(\alpha^{i+1} 2^{-1} r\right) \int_{\alpha^{i} 2^{-1} r}^{\alpha^{i+1} 2^{-1} r} A_{p}(\rho) d \rho\right) \\
& \leq C V_{p}(r)+\sum_{i=0}^{q-1}\left(\left((1+\epsilon)-\alpha^{i+1} 2^{-1}\right)^{-\nu}\left(V_{p}\left(\alpha^{i+1} 2^{-1} r\right)-V_{p}\left(\alpha^{i} 2^{-1} r\right)\right)\right) \\
& \leq C V_{p}(r)+\sum_{i=0}^{q-1}\left(\left((1+\epsilon)-\alpha^{i+1} 2^{-1}\right)^{-\nu}\left(C_{0} \alpha^{\nu}-1\right) V_{p}\left(\alpha^{i} 2^{-1} r\right)\right) \\
& \leq C V_{p}(r)\left(1+\sum_{i=0}^{q-1}\left((1+\epsilon)-(1+\epsilon / 2) \alpha^{i} 2^{-1}\right)^{-\nu}\right) \\
& \leq C V_{p}(r)\left(1+\int_{2^{-1} r}^{r}\left((1+\epsilon)-(1+\epsilon / 2) 2^{-1} \rho\right)^{-\nu} d \rho\right) \\
& \leq C V_{p}(r)\left(1+\epsilon^{-(\nu-1)}\right),
\end{aligned}
$$

and the lemma follows by combining this with (3).

Lemma 2. Let $K$ be a $k$-dimensional linear space of sections of a vector bundle $E$ over $M$. Assume that $M$ has volume growth at most of order $r^{\nu}$. Suppose each section $u \in K$ are polynomial growth of at most degree $d$. For $p \in M, \beta>1$, $\delta>0$, and $r_{0}>0$, there exists $r>r_{0}$ such that if $\left\{u_{i}\right\}_{i=1}^{k}$ is an orthonormal basis of $K$ with respect to the inner product $A_{\beta r}(u, v)=\int_{B_{p}(\beta r)}\langle u, v\rangle$, then

$$
\sum_{i=1}^{k} \int_{B_{p}(r)}\left|u_{i}\right|^{2} \geq k \beta^{-(2 d+\nu+\delta)} .
$$

Proof. For each $r>0$, let $A_{r}$ be the positive definite bilinear form defined on $K$ given by $A_{r}(u, v)=\int_{B_{p}(r)}\langle u, v\rangle$. Let us denote the trace of the bilinear form $A_{r}$ with respect to $A_{r^{\prime}}$ by $\operatorname{tr}_{r^{\prime}} A_{r}$. Similarly, let $\operatorname{det}_{r^{\prime}} A_{r}$ be the determinant of $A_{r}$ with respect to $A_{r^{\prime}}$. Assuming that the lemma is false, then for $r>r_{0}$, we have

$$
\operatorname{tr}_{\beta r} A_{r}<k \beta^{-(2 d+\nu+\delta)} .
$$

On the other hand, the arithmetic-geometric means asserts that

$$
\left.\left(\operatorname{det}_{\beta r} A_{r}\right)\right)^{1 / k} \leq k^{-1} \operatorname{tr}_{\beta r} A_{r} .
$$


This implies that

$$
\operatorname{det}_{\beta r} A_{r} \leq \beta^{-k(2 d+\nu+\delta)}
$$

for all $r>r_{0}$. Setting $r=r_{0}+1$ and iterating this inequality $j$ times yield

$$
\operatorname{det}_{\beta^{j} r} A_{r} \leq \beta^{-j k(2 d+\nu+\delta)} .
$$

However, for a fixed $A_{r}$-orthonormal basis $\left\{u_{i}\right\}_{i=1}^{k}$ of $K$, the assumption on $K$ and the volume growth assumption imply that there exists a constant $C>0$, depending on $K$, such that

$$
\int_{B_{p}(r)}\left|u_{i}\right|^{2} \leq C\left(1+r^{2 d+\nu}\right)
$$

for all $1 \leq i \leq k$. In particular, this implies that

$$
\operatorname{det}_{r} A_{\beta^{j} r} \leq k ! C \beta^{j k(2 d+\nu)} r^{k(2 d+\nu)} .
$$

This contradicts (5) as $j \rightarrow \infty$, and the lemma is proved.

Proof of Theorem 1. Let $\left\{u_{i}\right\}_{i=1}^{k}$ be an $A_{\beta r}$-orthonormal basis of any finite dimensional subspace $K \subset \mathcal{S}_{d}(M, E)$. Clearly, it suffices to prove the estimate for $k=\operatorname{dim} K$. Lemma 2 implies that there is a $r>0$ such that

$$
\sum_{i=1}^{k} \int_{B_{p}(r)}\left|u_{i}\right|^{2} \geq k \beta^{-(2 d+\nu+\delta)} .
$$

On the other hand, by setting $\beta=1+\epsilon$, Lemma 1 implies that

$$
\sum_{i=1}^{k} \int_{B_{p}(r)}\left|u_{i}\right|^{2} \leq m C \lambda \epsilon^{-(\nu-1)}
$$

because $\int_{B_{p}((1+\epsilon) r)}|u|^{2}=1$ for all $u \in\{\langle A, U\rangle\}$. The estimate on $k$ follows by setting $\epsilon=(2 d)^{-1}$ and observing that $\left(1+(2 d)^{-1}\right)^{-(2 d+\nu+\delta)}$ is bounded from below.

Note that Moser's iteration also works with functions satisfying $\Delta f \geq-g f$. In particular, if $g$ is a non-negative function with compact support, then (see [L 3]) $f$ will satisfy a mean value inequality for all $x \in M$ and for all $r>0$. This observation allow us to apply to harmonic sections of a bundle with curvature non-negative outside a compact set. We will now give a few corollaries of Theorem 1 by simply applying the corresponding Bochner-Weitzenböck formula to the harmonic sections. 
Corollary 2. Let $M^{n}$ be a complete manifold satisfying conditions $(W)$ and $(M)$. Then

$$
\operatorname{dim} \mathcal{H}_{d}(M) \leq C \lambda d^{\nu-1}
$$

In particular, if $M$ is quasi-isometric to a manifold with non-negative Ricci curvature, or $M$ is a minimal submanifold in Euclidean space with Euclidean volume growth, then

$$
\operatorname{dim} \mathcal{H}_{d}(M) \leq C d^{n-1}
$$

Corollary 3. Let $M$ be a manifold whose metric $d s^{2}$ is obtained by a compact perturbation of another metric $d s_{0}^{2}$ which has non-negative Ricci curvature. Suppose

$$
\mathcal{H}_{d}^{p}(M)=\left\{u \in \Lambda^{p}|\Delta u=0,| u \mid(x)=O\left(\rho^{d}(x)\right) \text { as } \rho \rightarrow \infty\right\}
$$

denotes the space of harmonic p-form of at most polynomial growth of degree $d$. Then

$$
\operatorname{dim} \mathcal{H}_{d}^{1}(M)=\operatorname{dim} \mathcal{H}_{d}^{n-1}(M) \leq n C d^{n-1} .
$$

If we further assume that $d s_{0}^{2}$ has non-negative curvature operator, then

$$
\operatorname{dim} \mathcal{H}_{d}^{p}(M) \leq\left(\begin{array}{c}
n \\
p
\end{array}\right) C d^{n-1}
$$

Corollary 4. Let $M$ be a complete Ricci flat manifold. Suppose $\mathcal{K}_{d}(M)$ is the space of Killing vector fields on $M$ which has polynomial growth of at most degree d. Then

$$
\operatorname{dim} \mathcal{K}_{d}(M) \leq C d^{n-1} .
$$

In the case of holomorphic sections of a Hermitian vector bundle over a Kähler manifold, we can modify the argument slightly by taking the appropriate Hermitian inner product on the fibers and using unitary transformations instead of orthogonal transformations in Lemma 1. In this setting, by apply the corresponding Bochner-Weitzenböck formula for holomorphic sections (see $[\mathrm{K}]$ for notation), Theorem 1 yields a Seigel type theorem.

Corollary 5. Let $M^{n}$ be a complete Kähler manifold of complex dimension $n$. Assume that $M$ satisfies conditions $(W)$ and $(M)$. Suppose $E$ is a rank-m Hermitian vector bundle over $M$ and that the mean curvature of $E$ is nonpositive. Let $\overline{\mathcal{H}}_{d}(M, E)$ be the space of holomorphic sections which is polynomial growth of at most degree $d$. Then

$$
\operatorname{dim} \overline{\mathcal{H}}_{d}(M, E) \leq m C \lambda d^{2 n-1} .
$$

It is clear that from the proof of Theorem 1, we actually proved a more general statement. 
Theorem 6. Let $M$ be a complete manifold satisfying condition $(W)$. Let $K$ be a linear space of sections of a rank-m vector bundle $E$ over $M$. Suppose each $u \in K$ satisfies the growth condition

$$
|u|(x)=O\left(\rho^{d}(x)\right)
$$

as the distance $\rho \rightarrow \infty$ to some fixed point $p \in M$, and the mean value inequality

$$
\lambda \int_{B_{x}(r)}|u|^{2} \geq V_{x}(r)|u|^{2}(x)
$$

for all $x \in M$ and $r>0$. Then there exists a constant $C>0$ depending only on $\nu$ and $C_{0}$, such that $\operatorname{dim} K \leq C m \lambda d^{\nu-1}$.

An example of this situation is when we consider a uniformly elliptic operator $L$ on $\mathbb{R}^{n}$. For instance, if $L$ is a uniformly bounded elliptic operator of divergence (or non-divergence) form, then Moser's iteration argument (Krylov's argument) [G-T] implies a mean value inequality for any non-negative subsolution of $L$. Therefore we can apply Theorem 6 to this setting. Before we state the next corollary, we would like to refer the reader to [A-Ln, Ln, Mr-S, Z] for previous work on a more restrictive class of uniformly elliptic operators on $\mathbb{R}^{n}$.

Corollary 7. Let $L=\frac{\partial}{\partial x^{i}}\left(a_{i j} \frac{\partial}{\partial x^{j}}\right)$ be an elliptic operator of divergence form with uniformly bounded coefficients defined on $\mathbb{R}^{n}$, i.e., there exists a constant $C_{1}>0$ such that $C_{1}^{-1}\left(\delta_{i j}\right) \leq\left(a_{i j}\right) \leq C_{1}\left(\delta_{i j}\right)$. Let

$$
\mathcal{H}_{d}(L)=\left\{u \in H_{1,2}^{\mathrm{loc}}\left(\mathbb{R}^{n}\right)|L(u)=0,| u \mid(x)=O\left(\rho^{d}(x)\right) \text { as } \rho \rightarrow \infty\right\}
$$

be the space of L-harmonic functions that has polynomial growth of degree at most d. Then

$$
\operatorname{dim} \mathcal{H}_{d}(L) \leq C d^{n-1} .
$$

Corollary 8. Let $L=a_{i j} \frac{\partial^{2}}{\partial x^{i} \partial x^{j}}$ be an elliptic operator of non-divergence form with uniformly bounded coefficients defined on $\mathbb{R}^{n}$, i.e., there exists a constant $C_{1}>0$ such that $C_{1}^{-1}\left(\delta_{i j}\right) \leq\left(a_{i j}\right) \leq C_{1}\left(\delta_{i j}\right)$. Let

$$
\mathcal{H}_{d}(L)=\left\{u \in H_{2, n}^{\text {loc }}\left(\mathbb{R}^{n}\right)|L(u)=0,| u \mid(x)=O\left(\rho^{d}(x)\right) \text { as } \rho \rightarrow \infty\right\}
$$

be the space of L-harmonic functions that has polynomial growth of degree at most d. Then

$$
\operatorname{dim} \mathcal{H}_{d}(L) \leq C d^{n-1} .
$$

Let us remark that the operator $L$ can be taken to be an elliptic system. One just need to ensure that if $U=\left(u_{1}, \ldots, u_{\ell}\right)$ is any solution to $L(U)=0$ then $|U|^{2}=\sum_{i} u_{i}^{2}$ will satisfy the mean value inequality. 


\section{References}

[A-Ln] M. Avellaneda and F. H. Lin, Une thèorème de Liouville pour des èquations elliptique à coefficients pèriodiques, C. R. Acad. Sci. Paris 309 (1989), 245-250.

[C-M 1] T. Colding and W. Minicozzi, On function theory on spaces with a lower Ricci curvature bound, Math. Res. Lett. 3 (1996), 241-246.

[C-M 2] - Harmonic functions with polynomial growth, preprint.

[C-M 3] _ Large scale behavior of kernels of Schrödinger operators, preprint.

[C-M 4]_, Generalized Liouville properties for manifolds, preprint.

[C-M 5] Harmonic functions on manifolds, preprint.

[C-M 6] W W Weyl type bounds for harmonic functions, preprint.

[C-M 7] , Liouville theorems for harmonic sections and applications, preprint.

[G-T] D. Gilbarg and N. S. Trudinger, Elliptic Partial Differential Equations of Second Order (2nd, ed.), Springer-Verlag, Berlin-Heidelberg-New York, 1983.

[G] A. Grigor'yan, The heat equation on noncompact Riemannian manifolds, Math. USSR Sbornik. 72 (1992), 47-77.

[K] S. Kobayashi, Differential Geometry of Complex Vector Bundles, Princeton Univ. Press, Princeton, 1987.

[L 1] P. Li, On the Sobolev constant and the p-spectrum of a compact Riemannian manifold, Ann. Scient. Éc. Norm. Sup. 4, T 13 (1980), 451-469.

[L 2] Lecture Notes on Geometric Analysis, Lecture Notes Series No. 6 - Research Institute of Mathematics and Global Analysis Research Center, Seoul National University, Seoul, 1993.

[L 3] _ Curvature and function theory on Riemannian manifolds, in preparation.

[Ln] F. H. Lin, Asymptotically conic elliptic operators and Liouville type theorems, preprint.

[M-S] J. H. Michael and L. Simon, Sobolev and mean-value inequalities on generalized submanifolds of $\mathbb{R}^{n}$, Comm. Pure Appl. Math. 26 (1973), 361-379.

[M-S] J. Moser and M. Struwe, On a Liouville-type theorem for linear and nonlinear elliptic differential equations on a torus, preprint.

[S-C 1] L. Saloff-Coste, Uniformly elliptic operators on Riemannian manifolds, J. Diff. Geom. 36 (1992), 417- 450.

[S-C 2] A note on Poincaré, Sobolev, and Harnack inequalities, Internat. Math. Res. Notices 2 (1992), 27-38.

[Y] S. T. Yau, Nonlinear Analysis in Geometry, L'Enseignement Mathématique, Série des Conférences de l'Union Mathématique Internationale, No. 8, SRO-KUNDIG, Genève, 1986.

[Z] L. Zhang, On generic eigenvalue flow of a family of metrics and its application in dimension estimates of polynomial growth harmonic functions, preprint.

Department of Mathematics, University of California Irvine, Irvine, CA 926973875

E-mail address: pli@math.uci.edu 